\title{
Correction to: Androgens and Anti-Müllerian Hormone in Infertile Patients
}

\author{
Panagiotis Drakopoulos ${ }^{1} \cdot$ Erlisa Bardhi $^{1} \cdot$ Sebastian Scherer ${ }^{1} \cdot$ Christophe Blockeel $^{1,2} \cdot$ Greta Verheyen $^{1}$. \\ Ellen Anckaert ${ }^{1} \cdot$ Herman Tournaye $^{1} \cdot$ Nikolaos P. Polyzos $^{3}$
}

Published online: 7 April 2021

(C) Society for Reproductive Investigation 2021

\section{Correction to: Reprod. Sci. https://doi.org/10.1007/s43032-021-00539-w}

This article was updated to correct author Nikolaos P. Polyzos's name.

Publisher's Note Springer Nature remains neutral with regard to jurisdictional claims in published maps and institutional affiliations.

Panagiotis Drakopoulos and Erlisa Bardhi are equally contributing first joint authors

The online version of the original article can be found at https://doi.org/ 10.1007/s43032-021-00539-w

Erlisa Bardhi

erlibardhi@gmail.com

1 Centre for Reproductive Medicine, Universitair Ziekenhuis Brussel, Vrije Universiteit Brussel, Laarbeeklaan 101,

1090 Brussels, Belgium

2 Department of Obstetrics and Gynaecology, University of Zagreb, Zagreb, Croatia

3 Department of Reproductive Medicine, Dexeus University Hospital, Salud de la Mujer Dexeus, Barcelona, Spain 